\title{
FishTracker: A GIS toolbox for kernel density estimation of animal home ranges that accounts for transit times and hard boundaries
}

\author{
$\underline{\text { Shawn W. Laffan }}^{\mathrm{a}, *}$, Matthew D. Taylor ${ }^{\mathrm{a}, \mathrm{b}}$ \\ ${ }^{a}$ School of Biological, Earth and Environmental Science, University of New South Wales, New South Wales, \\ 2052, Australia \\ ${ }^{b}$ Wild Fisheries Research, Port Stephens Fisheries Institute, Locked Bag 1, Nelson Bay, NSW, 2315, \\ Australia \\ Email: shawn.laffan@unsw.edu.au
}

\begin{abstract}
Understanding animal home ranges and other patterns of space utilisation is an important component of spatial ecology. It allows researchers to explore and explain site occupation and habitat preferences, and also interaction and avoidance behaviour. Conventional analyses of animal home ranges use points at which the animals are observed, sometimes weighted by the time difference between sequential observations. This creates an issue in that the analysis can assign undue weight to a sample point with a long time delay from the previous observation, as the full path from the preceding point is implicitly assigned to that point. Conventional analyses also do not take into account physical constraints such as boundaries (e.g. rivers, roads, cliffs) or the cost of traversing alternate possible paths between points, typically inferring a straight-line path between sequential samples. This conventional approach has obvious limitations, especially in constrained environments such as for fish in rivers and estuaries. These limitations can be attributed in large part to a lack of available software tools.
\end{abstract}

In this paper we describe a software tool we have developed that calculates kernel density estimates of occupation times by using the per-segment transit times along a path inferred using a cost surface that is constrained to remain between defined boundaries. To illustrate the functionality of the tool we use a data set of Argyrosomus japonicus (Mulloway) movements, an estuarine fish species, sampled from the Georges River estuary in Sydney, NSW. The approach is, however, generic and can be applied to any environment where animal movements are constrained, for example fragmented agricultural landscapes. The tool is written using the ESRI arcpy system, but is open source so can readily be ported to other GIS software packages.

Keywords: telemetry, estuary, river, Vemco Positioning System, home range, space use, GIS, spatial ecology 


\section{INTRODUCTION}

Least-cost path analysis is an analytical tool used to derive a cost-effective distance between an origin and destination. Least-cost approaches incorporate landscape properties to allow for more realistic representations of the connectivity between sites, and thus any spatial analyses using them (Laffan 2002; Lyon et al. 2010; Greenberg et al. 2011). Such approaches are employed in geospatial applications from landscape ecology (Adriaensen et al. 2003) to routing of roads or canals (Collischonn et al. 2000). In this paper we describe a software tool we have developed that employs least-cost path analysis to improve estimations of animal home ranges, using an example data set of fish locations obtained using acoustic telemetry in an estuary with complex meander geometry. While one cannot be certain the path taken by an animal was the one with the least cost, it nevertheless represents a more refined model of the path than a straight line.

Home ranges are the geographic areas that are typically occupied by individuals or groups of animals (e.g. packs, herds, flocks). Understanding animal home ranges and other patterns of space utilisation is an important component of spatial ecology. It allows researchers to explore and explain site occupation and habitat preferences, and also interaction and avoidance behaviour. For modelling purposes, "typical" home ranges are important for the calibration of dispersal kernels (Skalski et al. 2000; Rodríguez 2002; Radinger et al. 2013b) and of epidemiological models of disease spread (Laffan et al. 2011; Ward et al. 2013). They can also be used in the calibration of species distribution models such that the models include abiotic variables from across an animal's home range, rather than only from those locations where animals have been sampled (Stone et al. 2013).

As with any analysis and modelling problem, there are several ways of modelling animal home ranges (Downs et al. 2012; Scull et al. 2012). The most commonly used methods involve variations on either convex hulls or kernel density estimates. In the convex hull method, a polygon is fitted around the observation locations, constrained such that the angle of the lines at each vertex is convex outwards, i.e. there are no peninsulas or islands in the polygon outline. Variations on the convex hull generally allow islands or concave angles. Kernel density analyses pass a moving window analysis over the data, conventionally on a regular lattice such as a raster. For each location on the lattice the density of observations within a set radius is calculated, but where the contribution of each observation is weighted by its proximity to the centre of the moving window. Any location with a non-zero density is assumed to be occupied or used by the animal, and thus could be part of the home range. In both cases the area most commonly occupied is taken to be representative of a home range, for example the area that encompasses $90 \%$ or $50 \%$ of the observations or of the kernel density surface. The outline of these areas can be treated as space use contours.

Data for determining animal home ranges can be obtained from numerous sources. Common examples include radio tracking, satellite tags, GPS collars, and field observations. Acoustic telemetry and biotelemetry are modern approaches employed by fisheries scientists to monitor many aspects of fish biology, physiology, space-use and movement (Cooke et al. 2004; Cooke et al. 2005; Taylor et al. 2006; Walsh et al. 2013). Basic telemetry systems consist of a tag, implanted in a free-ranging aquatic animal, and a receiver to which data or signals from the tag are telemetered. Receivers can be deployed as automated fixedstations in an array configuration (passive tracking, Heupel et al. 2006), which can continually collect information from organisms that are within the range of a receiver. Receivers can also be portable (such as a boat-based receiver), and used to actively locate the position of a fish at a high spatial resolution (e.g. active tracking, Taylor et al. 2011). Modern telemetry approaches can also employ positioning systems (Vemco Positioning System, Espinoza et al. 2011), which use triangulation to combine the benefits of automated or passive tracking techniques with the high spatial resolution that is usually derived from active tracking approaches. Acoustic telemetry is now widely applied to study animals in all aquatic environments (e.g. oceanic, estuarine, riverine, lake), and this frequently produces large data sets with many hundreds or thousands of positional data points recorded for each fish (Payne et al. 2013; Walsh et al. 2013).

The derivation of movement rates and home ranges from positional data in complex meandering systems such as rivers and estuaries presents several problems. Firstly, current GIS-based calculations of step length (i.e. the distance between two successive data points) cannot account for the boundaries imposed by the water body shorelines for aquatic animals. Furthermore, migration corridors may be restricted to certain aquatic habitats or depth ranges. Secondly, the derivation of space-use contours using estimates of kernel density cannot be effectively constrained using shoreline features, and search radii and subsequent distributions frequently span non-aquatic areas. The latter issue is usually managed by clipping the resultant kernel density raster by the river- or estuary-feature. However, this may skew distributions, particularly where the meander length is small.

A second issue with data such as from telemetry relates to the time intervals between sequential observations. In many analyses, certainly those implemented using standard GIS tools, the observation points themselves are used in the home range analyses. Focusing on the points as the units of analysis implicitly ignores all 
points along the paths between sequential points, missing potentially important information. This effect increases as the distances between sequential observations increase. A more refined approach would model the least-cost path between pairs of sequential observations, and use points along this path in the analysis of the home range. This would also need to account for the time difference between observation pairs, calculating a transit time per geographic analysis unit (e.g. a raster cell).

An automated approach to address these issues when estimating spatial trends would improve the analysis of telemetry data, and add to the suite of software tools specifically designed to support spatial ecology research (e.g. Beyer et al. 2010). It is for these purposes that the FishTracker tool has been developed.

\section{MODEL DESCRIPTION}

Much of the FishTracker toolbox comprises standard data import and transformation tools. The key development is that it uses a least-cost path to determine travel velocity between sequential observations, converting this into per-segment transit times. These transit times are then used in a standard kernel density analysis (masked by the cost surface) to estimate an animal's home range.

The main steps of the FishTracker tool are:

1. Snap observation locations to the cost surface (Figure 1). This allows for coordinate imprecision and removes some of the data cleaning burden from the user.

2. For each observation $i$ in the set of observations $i=2$ to $n$

a. Calculate least-cost path distance to observation $i$ from observation $i-1$

b. Calculate time difference between observation $i$ and observation $i-1$

c. Calculate transit time per cell along the least-cost path as a linear allocation of the result of

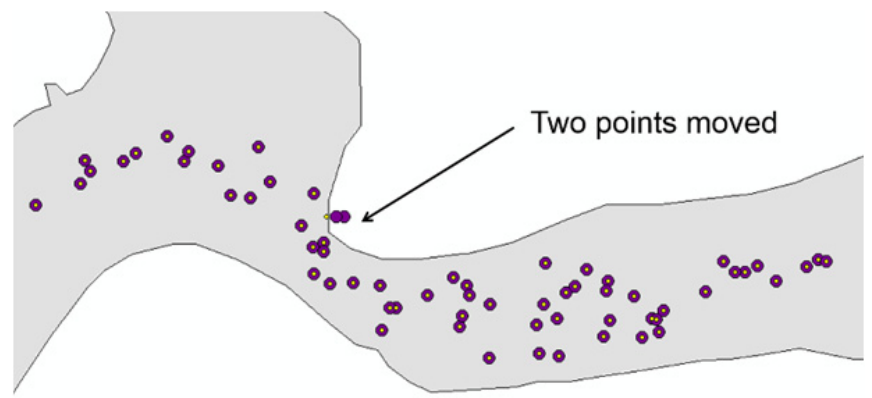

Figure 1. Points located on or outside the boundary of the cost surface raster are relocated to be one cell inside its outer edge. This ensures all least cost paths are valid. step $2 b$, and store as a raster.

3. Sum the transit rasters for all observations $i$ to generate an accumulated transit raster

4. Calculate a kernel density surface using the accumulated transit raster as the locations, weighted by their transit times. This is masked using the cost surface.

5. Calculate percentile surfaces of the kernel density surface excluding zero values (currently $50^{\text {th }}$ and $90^{\text {th }}$ percentiles).

The FishTracker model has been developed as an ArcPy toolbox for use with ArcGIS 10.1 (Figure 2). While ArcGIS is a proprietary system, the FishTracker code itself is open source (https://bitbucket.org/shawn_laffan/fish_tracker), with the key novelty written using SciPy functions, and can be readily ported to other GIS platforms.

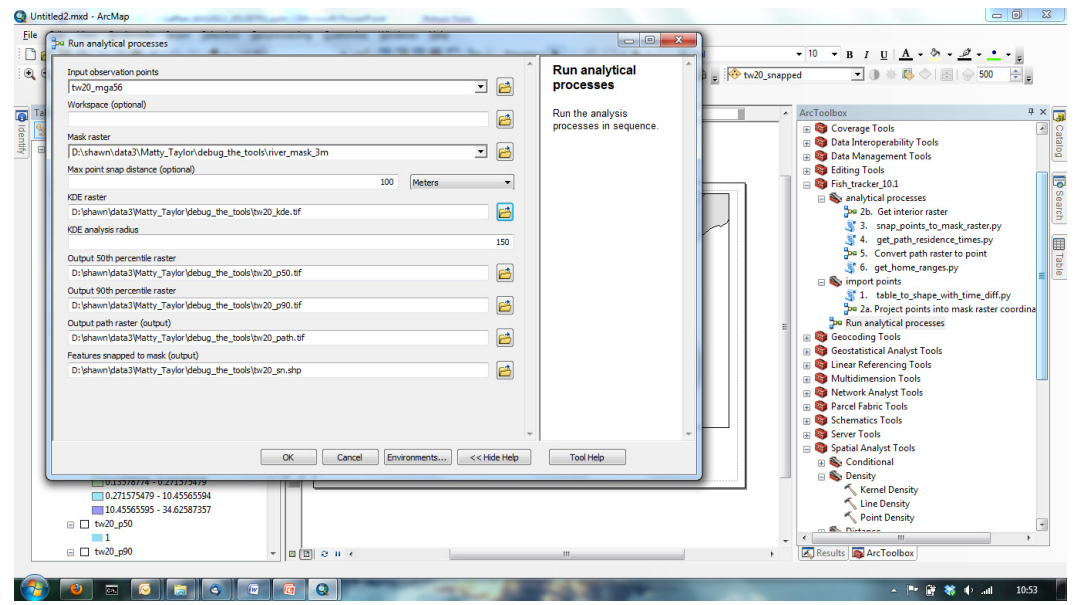

Figure 2. FishTracker is implemented as a toolbox within ArcGIS. 


\section{SAMPLE DATA COLLECTION AND ANALYSIS}

Eight fish were tagged and tracked in the Georges River estuary, Australia, continually for a period of 12 days. The Georges River is a riverine estuary located in the southern Sydney metropolitan area $\left(33.976^{\circ} \mathrm{S}\right.$, $\left.151.023^{\circ} \mathrm{E}\right)$, New South Wales, Australia (Figure 3). Mulloway (Argyrosomus japonicus; $42 \pm 2$ $\mathrm{cm}$, mean $\pm \mathrm{SE}$ ) were tagged with Sonotronics IBT-96-1 acoustic transmitters (Sonotronics, Arizona, USA) using conventional methods (see Taylor et al. 2013). Fish were continually monitored using a boat-based Sonotronics USR-96 ultrasonic receiver and $\mathrm{DH}-4$ hydrophone. Where a signal was located, an individual fish could be identified through

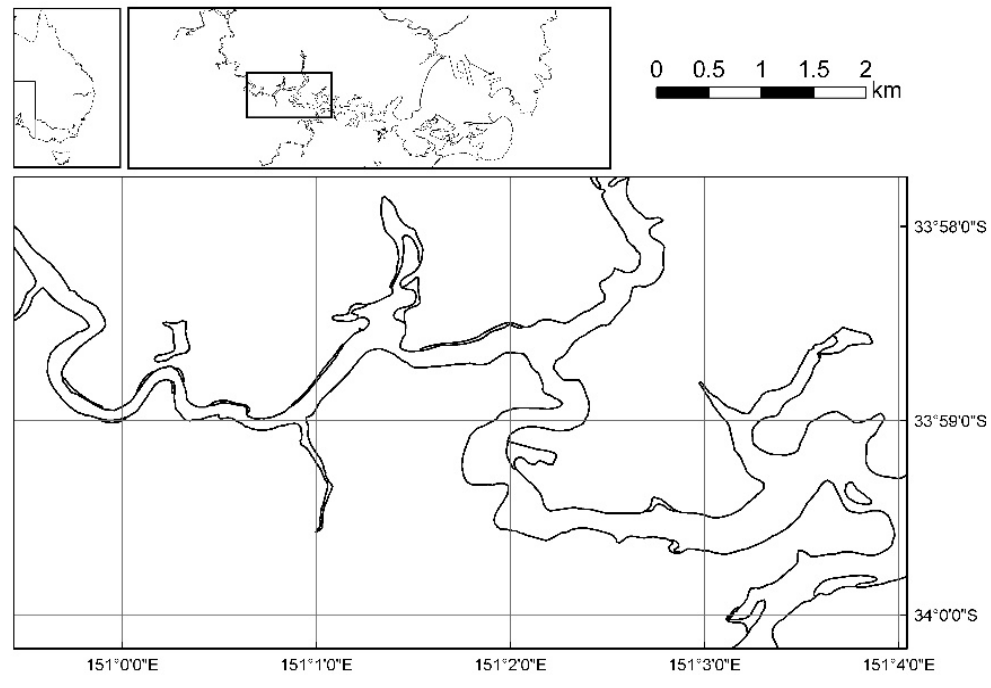

Figure 3. The example data are sampled from the Georges River estuary in Sydney, NSW, Australia. interpretation of an audible code.

The minimum range of the IBT-96-1 transmitters in this section of the Georges River was $324 \pm 12 \mathrm{~m}$ (mean $\pm \mathrm{SE}$ ) and repeated tracking studies have shown the location error to be $4.8 \pm 0.7 \mathrm{~m}$ (mean $\pm \mathrm{SE}$, Taylor et al. 2006). The tracking area was continually surveyed for signals by repeatedly travelling along the river, scanning all appropriate frequencies and, each time a fish was located, a GPS waypoint, time and depth were recorded. All spatial and temporal detection data were compiled in individual spreadsheet files for each fish, and imported into ArcGIS 10.1 for analysis. Data from a single fish are presented in this paper to demonstrate the FishTracker software tool.

Four analyses were conducted as a comparative analysis of the FishTracker approach with more conventional analyses. In all but one of the analyses the kernel surface was also masked to remain in the river, but was not itself weighted using least-cost path distances. The kernel bandwidth was set to $150 \mathrm{~m}$ following Taylor et al. (2006). Analysis 1 was an unweighted kernel density analysis of the observation points. Analysis 2 weighted the sample points by the time elapsed from the preceding sample point. Analysis 3 used a cost path surface weighted by transit times, but was unconstrained by the river (using a cost surface where all cells in the study area have a value of 1). Analysis 4 used the complete FishTracker approach. The cost path used was a raster of the Georges River at a $3 \mathrm{~m}$ resolution, with all cells assigned a value of 1 so there is no cost difference between deep water and shallow water. Its effect in this case is to constrain the paths to the river system.

\section{RESULTS AND DISCUSSION}

Conventional path and kernel density analysis using unweighted and unconstrained kernel density estimation highlights the issues encountered when using the kernel density tool in ArcGIS with fish movements in complex river systems (Figure 4a). Firstly, movement steps fail to account for the curvature of the river, and consequently cross land-water boundaries. Examples which use this conventional approach in the literature generally clip kernel density contours to the river or estuary feature (Figure 4b). This creates somewhat unrealistic contours which frequently create "islands" of space use, give higher weighting to areas near the shoreline than is expected from fish position data. This will consequently overestimate the spatial extent of home ranges, and also bias estimates of habitat use which are derived from them. Figure 4c demonstrates how these biases are overcome by incorporating the least-cost path in the kernel density estimate, with movement steps following the curvature of the river and space utilisation contours reflecting a more probable path taken by the fish that remains within the boundary of the aquatic feature. The modelled paths can however contain unusually abrupt turns. This is an interaction between the cost accumulation algorithm and a flat cost surface. Adding a small random component to the cost surface removes this effect and makes the resultant paths more linear in the absence of barriers (Figure 5). 

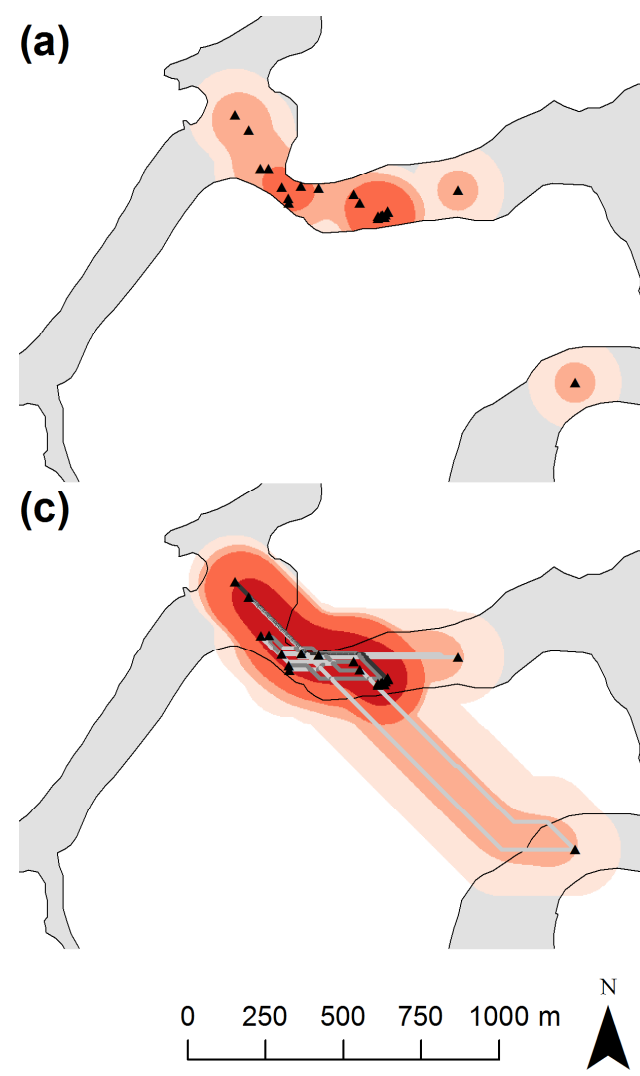

(b)

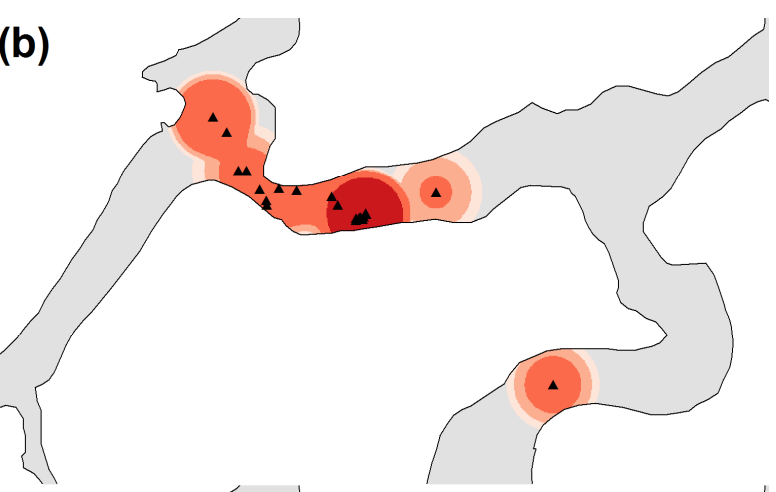

(d)

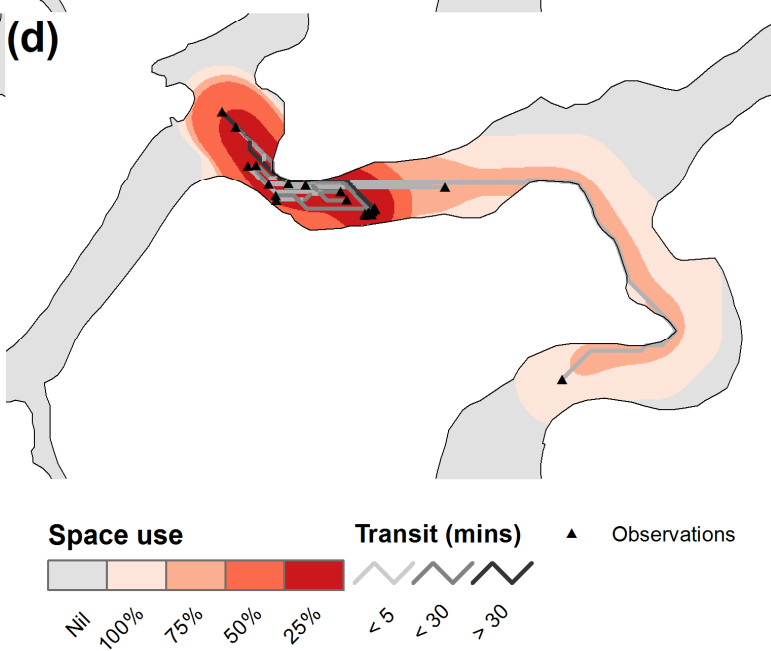

Figure 4. Home range estimates using different constraints. (a) Unweighted, no path constraints, masked to river. (b) As for (a), but weighted by time taken to reach sample location. (c) Kernel density of least cost path to reach sample location, unconstrained by river. Path segments are weighted by their transit times. (d)

The full FishTracker approach is as for (c) except the path is constrained to remain within the river.

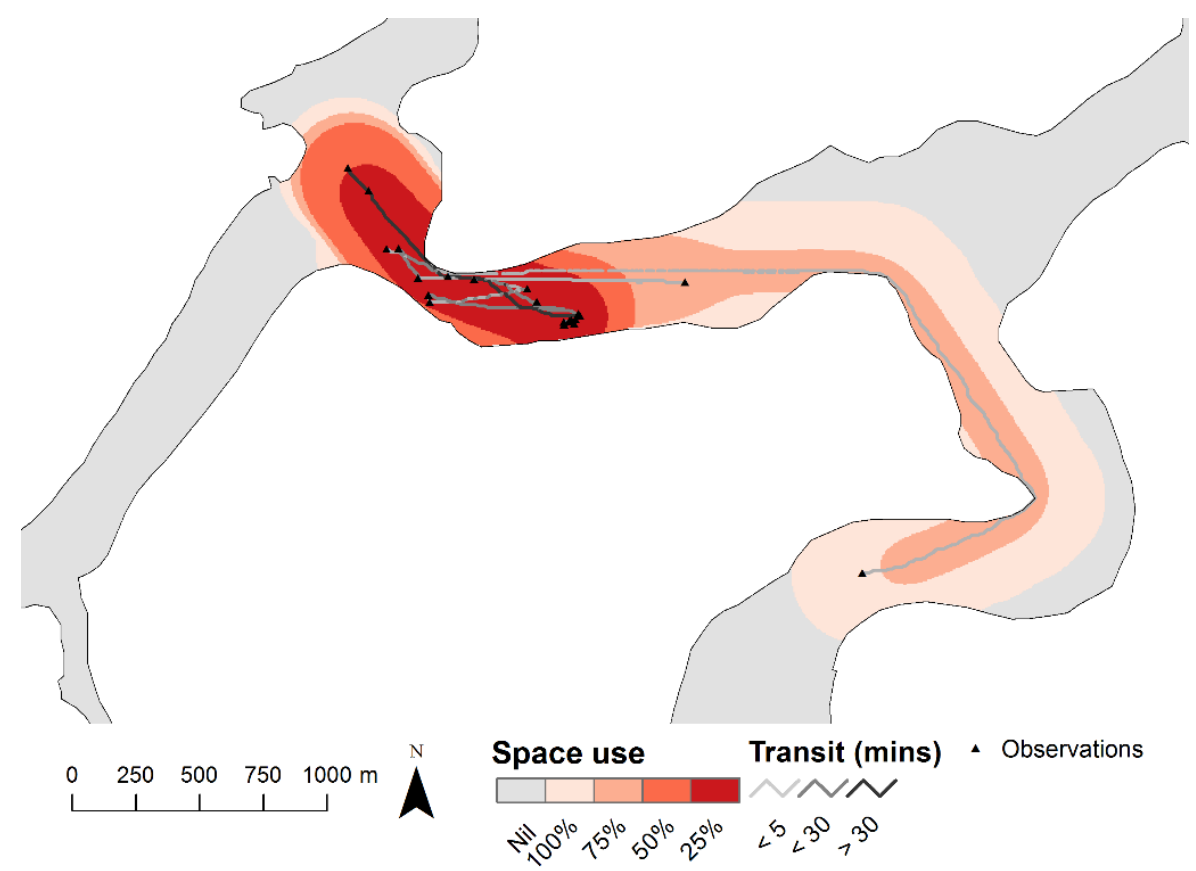

Figure 5. Randomly perturbing the cost surface by adding a small random component $(+/-5 \%$ in this case) removes the linear path effects, but makes only a small difference to the estimated home range shown in Figure 4d. 


\section{CONCLUSIONS AND FUTURE WORK}

The FishTracker software tool is implemented to work within the ESRI ArcGIS environment, but the code itself is open source, so the method can be ported to other GIS analysis environments (e.g. see Radinger et al. 2013a).

The underlying novelty of this software tool is the calculation and output of the distance between pairs of animal locations in aquatic environments of complex shoreline topography. This is a key advancement in the automation of analysis of animal movement data within set boundaries (such as the shoreline of an aquatic feature), and complements existing tools for analysis of animal movements. While development of this software tool was motivated by a need for analysis of fish telemetry data in such systems, it is equally applicable for any animal movement study, in water or on land, using any data which collect sequential records of animal locations. For example, one could analyse the movements of animals across a fragmented agricultural landscape where the cost surface represents the difficulty of moving within and between patches. It could also have applications in the analysis of population connectivity, for example genetic relatedness between patches of forest while also accounting for seed dispersal.

The FishTracker tool complements functionality of existing tools, such as the Geospatial Modelling Environment (GME), which while extremely useful for analysis of animal movement data (Beyer 2012), does not address the problems outlined in this paper. The incorporation of a weighted least-cost path calculation in the analyses shows additional promise for the analysis of large data sets derived from passive dispersed acoustic arrays (Heupel et al. 2006). Analysis of such datasets can rely on interpolation of movements between successive positions detected on different acoustic receivers, which in some cases are dispersed from other receivers (Pursche et al. 2013) or represent movements between entirely different arrays.

Future developments of the software tool will include a down-weighting factor to reduce the impact of large time differences between sequential samples. The kernel density analysis itself also currently uses Euclidean distances. While a path weighted variant could be implemented, for space-use estimation a linear estimate constrained by barriers should be sufficient for most purposes.

\section{ACKNOWLEDGEMENTS}

The authors wish to thank those that assisted during the tracking period, including I. Suthers, T. Mullaney, A. Pursche, A. Ferguson, H. El Hassan, E. Venstra, J. Smith and F. Ochwada, and K. Taylor for data entry. The authors also acknowledge the Australian Research Council (project LP0775000 AND LP100100367) and the NSW Recreational Fishing Saltwater Trust. Data collection from animals was authorised under UNSW Animal Research Authority 07/61A.

\section{REFERENCES}

Adriaensen F., J. Chardon, G. De Blust, E. Swinnen, S. Villalba, H. Gulinck \& E. Matthysen (2003) The application of 'least-cost' modelling as a functional landscape model. Landscape and urban planning, 64, 233-247.

Beyer H., J. Jenness \& S. Cushman (2010) Components of Spatial Information Management in Wildlife Ecology: Software for Statistical and Modeling Analysis. In: S. Cushman \& F. Huettmann (eds.) Spatial Complexity, Informatics, and Wildlife Conservation. Springer Japan.

Beyer H.L. (2012) Geospatial Modelling Environment. 0.7.1.0 ed.: http://www.spatialecology.com/gme.

Collischonn W. \& J.V. Pilar (2000) A direction dependent least-cost-path algorithm for roads and canals. International Journal of Geographical Information Science, 14, 397-406.

Cooke S.J., S.G. Hinch, M. Wikelski, R.D. Andrews, L.J. Kuchel, T.G. Wolcott \& P.J. Butler (2004) Biotelemetry: a mechanistic approach to ecology. Trends in Ecology \& Evolution, 19, 334-343.

Cooke S.J., G.H. Niezgoda, K.C. Hanson, C.D. Suski, F.J.S. Phelan, R. Tinline \& D.P. Philipp (2005) Use of CDMA Acoustic Telemetry to Document 3-D Positions of Fish: Relevance to the Design and Monitoring of Aquatic Protected Areas. Marine Technology Society Journal, 39, 31-41.

Downs J.A., J.H. Heller, R. Loraamm, D.O. Stein, C. McDaniel \& D. Onorato (2012) Accuracy of home range estimators for homogeneous and inhomogeneous point patterns. Ecological Modelling, 225, 66-73.

Espinoza M., T.J. Farrugia, D.M. Webber, F. Smith \& C.G. Lowe (2011) Testing a new acoustic telemetry technique to quantify long-term, fine-scale movements of aquatic animals. Fisheries Research, 108, 364-371.

Greenberg J.A., C. Rueda, E.L. Hestir, M.J. Santos \& S.L. Ustin (2011) Least cost distance analysis for spatial interpolation. Computers \& Geosciences, 37, 272-276. 
Heupel M.R., J.M. Semmens \& A.J. Hobday (2006) Automated acoustic tracking of aquatic animals: scales, design and deployment of listening station arrays. Marine and Freshwater Research, 57, 1-13.

Laffan S.W. (2002) Using process models to improve spatial analysis. International Journal of Geographical Information Science, 16, 245-257.

Laffan S.W., Z. Wang \& M.P. Ward (2011) The effect of neighbourhood definitions on spatio-temporal models of disease outbreaks: Separation distance versus range overlap. Preventive Veterinary Medicine, 102, 218-229.

Lyon S.W., R. Sørensen, J. Stendahl \& J. Seibert (2010) Using landscape characteristics to define an adjusted distance metric for improving kriging interpolations. International Journal of Geographical Information Science, 24, 723-740.

Payne N.L., D.E. van der Meulen, R. Gannon, J.M. Semmens, I.M. Suthers, C.A. Gray \& M.D. Taylor (2013) Rain reverses diel activity rhythms in an estuarine teleost. Proceedings Of The Royal Society B - Biological Sciences, 280, 20122363, http://dx.doi.org/10.1098/rspb.2012.2363.

Pursche A.R., I.M. Suthers \& M.D. Taylor (2013) Post-release monitoring of site and group fidelity in acoustically tagged stocked fish. Fisheries Management and Ecology, n/a-n/a.

Radinger J., J. Kail \& C. Wolter (2013a) FIDIMO - A free and open source GIS based dispersal model for riverine fish. Ecological Informatics.

Radinger J. \& C. Wolter (2013b) Patterns and predictors of fish dispersal in rivers. Fish and Fisheries, doi: $10.1111 /$ faf. 12028 .

Rodríguez M.A. (2002) Restricted movement in stream fish: The paradigm is incomplete, not lost. Ecology, $83,1-13$.

Scull P., M. Palmer, F. Frey \& E. Kraly (2012) A comparison of two home range modeling methods using Ugandan mountain gorilla data. International Journal of Geographical Information Science, 26, 2111-2121.

Skalski G.T. \& J.F. Gilliam (2000) Modeling diffusive spread in a heterogeneous population: A movement study with stream fish. Ecology, 81, 1685-1700.

Stone O.L., S. Laffan, D. Curnoe \& A.R. Herries (2013) The Spatial Distribution of Chacma Baboon (Papio ursinus) Habitat Based on an Environmental Envelope Model. International Journal of Primatology, 34, 407-422.

Taylor M.D., A.V. Fairfax \& I.M. Suthers (2013) The race for space: Using acoustic telemetry to understand density-dependent emigration and habitat selection in a released predatory fish. Reviews in Fisheries Science, DOI: 10.1080/10641262.2013.796813.

Taylor M.D. \& A. Ko (2011) Monitoring acoustically tagged king prawns Penaeus (Melicertus) plebejus in an estuarine lagoon. Marine Biology, 158, 835-844.

Taylor M.D., S.D. Laffan, D.S. Fielder \& I.M. Suthers (2006) Key habitat and home range of mulloway (Argyrosomus japonicus) in a south-east Australian estuary: Finding the estuarine niche to optimise stocking. Marine Ecology-Progress Series, 328, 237-247.

Walsh C.T., I.V. Reinfelds, M.C. Ives, C.A. Gray, R.J. West \& D.E. van der Meulen (2013) Environmental influences on the spatial ecology and spawning behaviour of an estuarine-resident fish, Macquaria colonorum. Estuarine, Coastal and Shelf Science, 118, 60-71.

Ward M.P., B.D. Cowled, F. Galea, M.G. Garner, S.W. Laffan, I. Marsh, K. Negus, S.D. Sarre \& A.P. Woolnough (2013) Salmonella infection in a remote, isolated wild pig population. Veterinary Microbiology, 162, 921-929. 